\title{
Nuevas citas de flora amenazada y rara en el País Vasco II.
}

\section{New records of threatened and rare flora in the Basque Country II.}

\author{
Juan Manuel Pérez de Ana ${ }^{1}$
}

A la memoria de Carlos Aseginolaza Iparragirre

\section{Resumen}

Se ofrecen 57 citas interesantes desde el punto de vista corológico de 43 plantas amenazadas y raras en el País Vasco (Bizkaia y Araba); 10 de ellas están protegidas en la Comunidad Autónoma del País Vasco.

Palabras clave: Álava-Araba, Bizkaia, conservación de la biodiversidad, especies amenazadas.

\begin{abstract}
57 observations of chorological interest of 43 threatened and rare plants in the Basque Country (Biscay and Araba) are offered; 10 of these plants are protected in the Autonomous Region of the Basque Country.
\end{abstract}

Key words: Álava-Araba, Bizkaia, biodiversity conservation, species of concern.

\section{Laburpena}

Honakoan, Euskal Herriko (Bizkaia eta Araba) 43 landare mehatxatu eta arraroren 57 aipamen interesgarri, korologiaren ikuspuntutik; horietatik 10 babestuta daude Euskal Autonomia Erkidegoan.

Gako hitzak: Álava-Araba, Bizkaia, biodibertsitatearen kontserbazioa, espezie mehatxatuak.

1 Sociedad de Ciencias Naturales de Sestao / Sestaoko Natur Zientzien Elkartea 
Se añaden 57 citas nuevas de 43 especies de flora amenazada y rara en el País Vasco (Bl: Bizkaia y VI: Araba), 10 de ellas incluidas en el Catálogo Vasco de Especies Amenazadas de la Comunidad Autónoma del País Vasco (Orden de 10 de enero de 2011), obtenidas en el período 2013-2016. Una es primera cita en la comunidad autónoma del País Vasco: Rhinanthus minor L. y otra en Bizkaia: Campanula rapunculus L. En su caso, se ofrecen los datos obtenidos del censo de sus poblaciones con el fin de que sirvan a su conservación. Para cada cita señalamos: municipio, paraje, cuadrícula UTM 1X1 km en Datum ETRS89, altitud, hábitat y fecha. Cuando se indica "Herbario SESTAO" significa que se conservan pliegos en el herbario SESTAO (Sociedad de Ciencias Naturales de Sestao). Todas las citas están incluidas en el huso 30T y, más en concreto, en 9 cuadrículas UTM de $10 \times 10$ km: VN86, VN95, VN96, WN06, WN07, WN08, WN16, WN18 y WN26. Se ha proporcionado al Servicio de Patrimonio Natural del Departamento de Medio Ambiente de la Diputación Foral de Bizkaia información geográfica de alta precisión de los hallazgos. Se ha seguido la nomenclatura de Flora iberica (www.floraiberica.es) y, en su defecto, la del Sistema de información sobre las plantas de España (www.anthos.es).

\section{NUEVAS CITAS}

\section{Allium schoenoprassum $\mathrm{L}$.}

(VI) Ayala, Peña del Aro, VN8864, 1.110 m, población de unos 3 metros de longitud en una grieta orientada al suroeste cerca de la cima, 14.07.2016.

Plantas de hojas glabras, cilíndricas y fistulosas, con tépalos purpúreos de 8 a $11 \mathrm{~mm}$ de longitud (Aedo, 2013). Primera cita de Sierra Sálvada y en la vertiente cantábrica de la comunidad autónoma del País Vasco, mientras que Alejandre et al. (2006) la citaron de varias localidades del burgalés Valle de Losa.

\section{Asplenium septentrionale (L.) Hoffm. subsp. septentrionale}

(BI) Orozko, monte Untzeta, WN0776, 690 m, 9 plantas en grietas verticales de una roca silícea orientada al este, 09.05.2014. Incluido en la categoría "vulnerable" del Catálogo Vasco de Especies Amenazadas.

Aseginolaza et al., (1985) la citaron en Bizkaia: monte Ganekogorta, WN0183, 950 m; Orozko: monte Untzeta, WN0873, 430 m; y en Álava: Landeta, VN9982, 800 m. Ya lo citamos de los montes Gallarraga y Ganekogorta, siendo frecuente en este último (Pérez de Ana, 2014). Carlos Aseginolaza (com. pers.) la encontró en el monte Untzeta durante el trabajo de campo realizado en 1982-1984 para el "Catálogo florístico de Álava, Vizcaya y Guipúzcoa". Tras varios intentos infructuosos, la reencontramos. 


\section{Astragalus glycyphyllos L.}

(BI) Orduña, monte Atxondo, VN9862 470 m, borde de quejigal, 11.06.2015.

Segunda cita de Orduña. Tras buscarla infructuosamente en VN9760, 450 m (Aseginolaza et al., 1985), aparece no lejos de allí. Dado que entonces no se disponía de geoposicionamiento, puede que se trate de un error en la asignación de la cuadrícula de 1 km.

\section{Baldellia ranunculoides (L.) Parl.}

(VI) Amurrio, Bigandi, WN0360, 670 m, borde de charca, 12.06.2014.

Plantas con numerosas inflorescencias con más de 7 flores y frutos con más 30 aquenios (Talavera et al., 2010). Primera cita en Sierra Sálvada y su entorno, las citas más cercanas son del Gorbea (Aseginolaza et al., 1985).

\section{Berberis vulgaris L. subsp. vulgaris}

(BI) Orozko, monte Usotegieta, WN1566, 1.180, población de 8×3 metros en el karst, 25.08.2016.

Nueva población que supone la segunda cita del Macizo del Gorbeia, descubierta por Joserra Undagoitia. Incluido en la categoría "rara" del Catálogo Vasco de Especies Amenazadas.

\section{Botrychium lunaria (L.) Sw.}

(BI) Zeanuri, Arimegorta, WN1966, 880 m, 12 plantas fértiles en pasto montano, 23.06.2016. Tras la primera cita en Bizkaia: monte Aldamin, (Aseginolaza et al., 1985), Patino et al., (20092010) añadieron seis localidades cercanas, aunque ninguna en esta cuadrícula, en la que se halla a una altitud menor que las conocidas. Descubierta por Joserra Undagoitia. Incluido en la categoría "rara" del Catálogo Vasco de Especies Amenazadas.

\section{Campanula rapunculus $\mathrm{L}$.}

(BI) Orduña, Lendoño de Abajo, VN9762, 350 m, herbazal a pie de carretera, 11.06.2015. Herbario SESTAO 23641.

Lázaro (Gredilla, 1913: 96) era el único que había citado esta planta de Bizkaia, en Berango, cita que Aseginolaza et al. (1985: 735) consideraron muy dudosa y que no se ha ratificado desde entonces.

\section{Carex cuprina (I. Sándor ex Heuff.) Nendtv. ex A. Kern.}

(VI) Amurrio, Bigandi, WN0360, 670 m, borde de charca, 12.06.2014.

Plantas cespitosas con vainas de color pardo claro, tallos escábridos y trígonos con ángulos ligeramente alados, con varias espigas similares y flores con dos estigmas (Luceño, 2007). Citada de Orduña, VN9959, 280 m (Aseginolaza et al., 1985). Segunda cita de Sierra Sálvada y su entorno. 


\section{Chamaespartium sagittale (L.) P. E. Gibbs}

(VI) Ayala, Sima del Avellano, VN9164, pasto montano, 26.06.2014.

Dentro de Sierra Sálvada solo se conocía de (BI) Orduña, VN9659, 1.000 m (Aseginolaza et al., 1985). En la parte burgalesa del monte Txarlazo es bastante común (obs. pers.). Esta es la segunda cita en el territorio vasco de Sierra Sálvada y la primera en su parte alavesa.

\section{Cirsium oleraceum (L.) Scop.}

(VI) Legutiano, ermita de Santa Cristina, WN2562, 570 m, talud de carretera rezumante y sombrío, 21.08.2016.

En el Macizo del Gorbeia se conocía de Igorre (Guinea, 1953) y Orozko, Itzina, WN1470, 1.000 m (Patino \& Valencia, 1989). Tercera cita y primera de su parte alavesa. Descubierta por Javier Fernández y comunicado por Joserra Undagoitia.

\section{Coronilla glauca L.}

(BI) Orduña, La Rondina, VN9859, 310 m, varias plantas ocupando 150 metros cuadrados sobre suelo removido y baldío, en plena floración, 19.01.2016. Herbario SESTAO 23643.

En Bizkaia solo se conocía de Leioa, WN09, 70 m, de procedencia incierta, en terreno removido (Aseginolaza et al., 1985). Primera cita de Sierra Sálvada y segunda de Bizkaia, probablemente también de origen antrópico.

\section{Dactylorhiza fuchsii (Druce) Soó}

(BI) Zeanuri, Atxuri, WN2065, 770-880 m, taludes rezumantes, 13.06.2015.

Argüelles et al. (2004-2005) la identificaron en un pliego de herbario (ARAN 09355) que recogieron los autores del "Catálogo florístico de Álava, Vizcaya y Guipúzcoa" en Ereño (BI), Bustanigan, WN3399, 500 m, 26.06.1982, Hermosilla \& Sabando (1993) la citaron por primera vez del País Vasco, dando cinco localidades de Álava y una de Bizkaia, Gorbea, aunque es ese mismo pliego de herbario el que sirve para citarla del Gorbea alavés. Segunda cita del Macizo del Gorbeia y tercera de Bizkaia. Población descubierta por Joserra Undagoitia.

\section{Doronicum carpetanum Boiss. \& Reut. ex Willk. \& Lange subsp. pubescens (Pérez Morales, Penas, Llamas \& Aedo) Aizpuru}

(BI) Orozko, Errekagatxo, WN1267, 660 m, barranco húmedo orientado al norte, 02.06.2015.

(BI) Arrankudiaga, Urdiola, WN0776, 570-580 m, claros forestales en ladera orientada al norte, 12.05.2016.

Plantas con hojas con abundante indumento como es característico de esta subespecie (Pérez Morales et al., 1994), como todas las plantas estudiadas desde el oeste de Cantabria 
hasta el Pirineo occidental (Aizpuru et al. 1998). En el Macizo del Gorbeia se conocía de dos localidades en su zona alavesa: Zigoitia, monte Oketa, WN26, 900 m, y Asunkorta, WN1862, 1.200 m (Aseginolaza et al., 1985). Tercera población del Macizo del Gorbeia, la primera de la parte vizcaína. Dos grandes poblaciones, una con 96 tallos con flores en Orozko y otra, comunicada por Dani Guillegui, con 122 tallos con flores en Arrankudiaga, en la ladera norte del monte Untzeta.

\section{Epipactis viridiflora Hoffm. ex Krock}

(BI) Zeanuri, Lanbreabe, WN1867, 640 m, 6 plantas florecidas en un claro de hayedo maduro, 21.08.2016.

Plantas con hojas más cortas que los entrenudos y flores con sépalos y pétalos netamente verdes y epiquilo anchamente cordiforme (Crespo, 2005). Hemos vuelto a encontrar la única cita de Bizkaia que dieron Hermosilla \& Sabando (1998) con el nombre de Epipactis purpurata. Si solo había 2 ejemplares en 1998, 18 años después contamos 6 plantas. Comunicada por Joserra Undagoitia.

\section{Euphorbia hyberna L. subsp. hyberna}

(VI) Amurrio, Tertanga, VN9657, 840 m, repisa herbosa, 05.05.2016.

Dentro de Sierra Sálvada se conocía del municipio alavés de Ayala (Aseginolaza et al., 1985) y del vizcaíno de Orduña (Pérez de Ana, 2007-2008 y 2009-2010, y Patino et al., 2009-2010). Aportamos la primera cita para el municipio de Amurrio dentro de Sierra Sálvada.

\section{Festuca paniculata (L.) Schinz \& Thell. subsp. Iongiglumis (Litard.) Kerguélen}

(BI) Orduña, monte Txarlazo, VN9658, 900 m, repisa herbosa al pie del cantil, 18.05.2015.

Plantas con densas macollas y cañas altas, hojas de lámina en V abierta, panículas con una o dos ramas en el nudo inferior y espiguillas doradas de 15-17 mm de longitud (Llamas et al., 2002). Segunda cita de Bizkaia tras la de Carranza, monte Zalama, VN6677, 650 m y segunda de Sierra Sálvada tras la de Ayala, monte Eskutxi, VN8965, 1.000 m (Aseginolaza et al., 1985).

\section{Galium odoratum (L.) Scop.}

(BI) Zeanuri, Atxuri, WN2065, 790 m, hayedo viejo y trasmocho entre bloques calizos, 13.06.2015. Herbario SESTAO 23642.

Del Macizo del Gorbeia existía una cita antigua (Gandoger, 1917: 149), que corroboraron Patino et al. (2009-2010), al tiempo que desechaban la cita de Emilio Guinea en Bakio. Tercera cita por lo tanto del Macizo del Gorbeia y de Bizkaia; densa población en una parcela de unas 7 hectáreas de hayedo. 


\section{Himantoglossum hircinum (L.) Spreng.}

(VI) Ayala, Izoria, VN9666, 270 m, un ejemplar en una cuneta, 14.05.2014

(BI) Orduña, La Rondina, VN9859, 290 m, un ejemplar en una cuneta, 14.05.2015.

Llorente et al., (2009) la citaron por primera vez de Bizkaia: Getxo, La Galea, VP9701, 80 m. Aportamos la primera cita de la comarca cantábrica alavesa y la segunda de Bizkaia, esta última comunicada por Michael Anderson. Incluido en la categoría "rara" del Catálogo Vasco de Especies Amenazadas.

\section{Huperzia selago (L.) Bernh. ex Schrank \& Mart. subsp. selago}

(BI) Orozko, monte Oderiaga, VN1266, 1.230 m, repisa en roquedo silíceo, 09.01.2016.

Uribe-Echebarría (1982) la citó del monte Oderiaga, WN1366, 1.300 m, grietas de rocas silíceas. Tras buscarla infructuosamente en dicha cuadrícula UTM 1×1 Km, aparece en una colindante, tal vez, como hemos mencionado antes, por error en la asignación de cuadrícula. La cima del monte Oderiaga solo alcanza 1.244 metros. Incluido en la categoría "rara" del Catálogo Vasco de Especies Amenazadas.

\section{Iberis carnosa Willd.}

(Bl) Orozko, Atxaragun, WN1469, 800 m, roquedo calizo, 25.06.2013.

(BI) Zeanuri, monte Lekanda, WN1668, 1.200 m, roquedo calizo, 14.06.2015.

(BI) Orozko, Lapurzulo, WN1565, 880 m, lastras calizas junto a arroyo, 09.05.2016.

Segunda, tercera y cuarta citas del Macizo del Gorbeia tras la de Zeanuri, monte Aldamin, WN1865, 1.320 m (Aseginolaza et al., 1985).

\section{Isopyrum thalictroides $\mathrm{L}$.}

(BI) Areatza, Pagomakurra, WN1669, 960 m, población de 600 metros cuadrados en hayedo aclarado pedregoso, 11.05.2016.

Citada de Zigoitia, Mairuelegorreta, WN2063, 1.000 m (Uribe-Echebarría, 1981), Orozko, Ibarra, WN1272, 280 m, y Zeanuri, Igiriñao, WN1665, 960 m (Aseginolaza et al., 1985). Cuarta población del Macizo del Gorbeia, donde es una especie escasa.

\section{Limodorum abortivum (L.) Sw.}

(Bl) Orduña, La Antigua, VN9760, 400 m, borde de quejigal, 08.05.2014.

(VI) Ayala, Menoio, VN9468, 410 m, cuneta, 28.05.2014.

En Bizkaia se conocía de Mañaria, Mugarra, WN2677, 600 m (Aseginolaza et al., 1985). Desconocida de Sierra Sálvada y su entorno. Aportamos la segunda cita de Bizkaia y la primera de la comarca cantábrica alavesa. En Orduña conté 27 tallos en el año 2014 y 16 en 2015. La población de Ayala, comunicada por Goretti Mendia, estaba compuesta por 
10-12 tallos unos días antes. Tras el paso de una máquina segadora por la cuneta, desaparecieron la mitad de esos tallos.

\section{Marrubium vulgare L.}

(VI) Ayala, Maroño, VN9566, 360 m, terreno baldío en el pueblo, 05.05.2016.

En Sierra Sálvada y su entorno se conocía de Orduña, VN9960, 270 m (Aseginolaza et al., 1985), donde la hemos buscado infructuosamente en los últimos años. Encontramos una segunda localidad, la primera de la comarca cantábrica alavesa. Pocos días después de su descubrimiento la población fue segada.

\section{Moricandia arvensis (L.) DC.}

(BI) Orduña, La Rondina, VN9859, 320 m, terrenos removidos y baldíos, 19.01.2016. Herbario SESTAO 23640.

Después de la cita de Barredo (2005) en Bilbao, esta es la segunda cita de Bizkaia y la primera de Sierra Sálvada y su entorno.

\section{Muscari neglectum Guss. ex Ten.}

(BI) Orduña, Lendoño de Arriba, VN9563, 490 m, grupo de 6 plantas en pasto erosionado orientado al sur, 19.04.2014.

Se conocía de Orduña (Aseginolaza et al., 1985). Segunda localidad de Bizkaia y de Sierra Sálvada.

\section{Ophioglossum vulgatum L.}

(BI) Zeanuri, londegorta, WN1866, 740 m, unos 100 helechos sobre suelo permanentemente muy húmedo debido a filtraciones que se producen en la cubeta de un pequeño embalse, 13.06.2015.

Se conocía de Zeanuri, Barazar, WN26, 604 m (Onaindia, 1986) y de Zeanuri, Igiriñao, WN1766, 1070 m (Patino et al., 2009-2010). Tercera población del Macizo del Gorbeia, descubierta por Joserra Undagoitia. A diferencia de los diminutos ejemplares de Igiriñao, los ejemplares de londegorta alcanzan el tamaño habitual en la especie. Incluido en la categoría "rara" del Catálogo Vasco de Especies Amenazadas.

\section{Ophrys aveyronensis (J. J. Wood) P. Delforge}

(VI) Amurrio, Tertanga, VN9758, 470 m, un único ejemplar en pastos erosionados, 15.06.2014. (Fig 1).

Aseginolaza et al., 1985 la citaron como O. arachnitiformis de Orduña, VN9760, 400 m, luego identificada como perteneciente a esta especie a partir del pliego de herbario y fotografías 


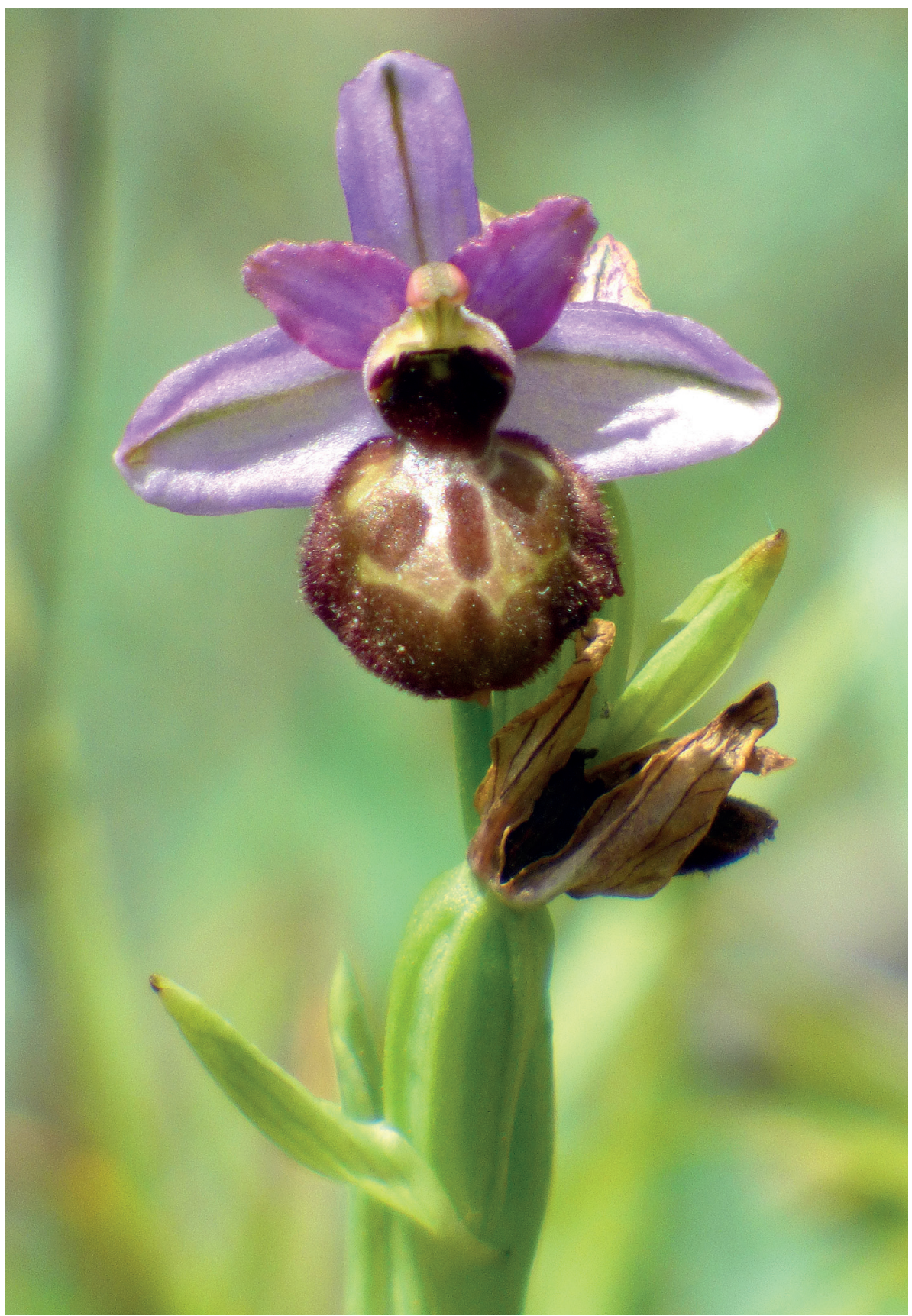

Fig. 1.- Flores de Ophrys aveyronensis, fotografiado en Amurrio.

Fig. 1.- Flowers of Ophrys aveyronensis, photographed in Amurrio. 
(Hermosilla \& Sabando, 1998). En su única localidad de Bizkaia la hemos buscado varios años sin llegar a localizarla de nuevo. De Álava está citada de Bernedo (Uribe-Echebarría, 2001) y Valdegovía (Silván et al., 2000). Cuarta localidad del País Vasco y segunda de la parte vasca de Sierra Sálvada. Incluido en la categoría "vulnerable" del Catálogo Vasco de Especies Amenazadas.

\section{Orchis coriophora L.}

(BI) Orduña, Lendoño de Arriba, VN9562, 480 m, un único ejemplar en pasto-enebral con junquillo, 03.06.2016.

Cuarta cita vizcaína y tercera orduñesa (Pérez de Ana, 2014).

\section{Oreochloa confusa (Coincy) Rouy}

(VI) Amurrio, Tertanga, VN9657, 850 m, borde del cantil, 05.05.2016.

Citada de Orduña, VN9659, 1.000 m (Aseginolaza et al., 1985). Segunda localidad de Sierra Sálvada y primera de su parte alavesa.

\section{Paris quadrifolia $\mathrm{L}$.}

(BI) Zeanuri, Atxuri, WN2065, 790 m, 56 plantas, solo una florecida, en hayedo viejo y trasmocho entre bloques calizos, 13.06.2015.

Citada de Zeanuri, monte Aldamin WN1865, 1350 m, y Zeanuri, Atxuri, WN2165, 900 m (Aseginolaza et al., 1985). En la última localidad la hemos buscado sin localizarla, pero Joserra Undagoitia descubrió el 24 de mayo de 2014 esta tercera población del Macizo del Gorbeia. Incluido en la categoría "rara" del Catálogo Vasco de Especies Amenazadas.

\section{Pedicularis tuberosa $\mathrm{L}$.}

(VI) Ayala, portillo de las Escaleras, VN9164, 1.000 m, repisa herbosa, 26.06.2014.

Citada de Ayala, monte Eskutxi, VN8965, 1.000 m (Aseginolaza et al., 1985). Segunda localidad de Sierra Sálvada. Incluido en la categoría "vulnerable" del Catálogo Vasco de Especies Amenazadas.

\section{Pinguicula lusitanica L.}

(BI) Orozko, Lapurruntxitako Atxa, WN0968, 700 m, cientos de plantas en una turbera de 15×10 metros, 10.06.2016.

(BI) Orozko, Algorta, WN1367, 850 m, cabecera de una gran turbera, 26.06.2016.

Citada de Zeanuri, puerto de Barazar, WN2368, 620 m, y Zigoitia, Murua, WN2060, 800 m (Aseginolaza et al., 1985). Tercera y cuarta población del Macizo del Gorbeia, la de Lapurruntxitako Atxa descubierta por Joserra Undagoitia y la de Algorta comunicada por Jon Fernández Pérez. Incluido en la categoría "rara" del Catálogo Vasco de Especies Amenazadas. 


\section{Polygonatum multiflorum (L.) All.}

(BI) Artea, Mendigana, WN1572, 620 m, 7 plantas en el talud de la pista que sube de Areatza a Pagomakurra, 27.05.2015.

(BI) Artea, Pagomakurra, WN1669, 880 m, 38 plantas sobre arroyo, cerca del aparcamiento, 27.05.2015.

(BI) Zeanuri, Arimegorta, WN1966, 860 m, 5 plantas sin flores al pie del cantil, 23.06.2016.

(BI) Zeanuri, Otzarreta, WN2365, 630 m, 2 plantas sin flores en talud de arroyo, 23.06.2016. Se conocía de Zeanuri, Arraba (Onaindia, 1986), Zeanuri, monte Aldamin, WN1865, 1.150 m (Aseginolaza et al., 1985) y Orozko, Atxaragun, WN1469, 940 m (Pérez de Ana, 2014). Otras cuatro poblaciones del Macizo del Gorbeia, la de Mendigana descubierta por Jesús Mari Molledo y las otras tres por Joserra Undagoitia.

\section{Potamogeton lucens L.}

(VI) Ayala, embalse de Maroño, VN9565, 310 m, orilla fangosa, 08.06.2016.

Se conocía de tres embalses de Gipuzkoa (Aizpuru et al., 1996 y Campos et al., 2002); y del alavés embalse de Ullibarri (Aseginolaza et al. 1985). La encontramos en un segundo embalse alavés.

\section{Ranunculus aquatilis L.}

(VI) Ayala, embalse de Maroño, VN9565, 310 m, orilla fangosa, 08.06.2016.

Citada del embalse de Ullibarri (Aseginolaza et al., 1985). Además, consultando el portal de GBIF puede verse que en el herbario VIT se conservan pliegos herborizados en balsas de Badaya y en una charca de Hueto Abajo. Lo encontramos en un segundo embalse del País Vasco.

\section{Ranunculus parviflorus L.}

(VI) Ayala, monte Ungino, VN9264, 710 m, cientos de plantas en reposaderos de ganado sobre pasto montano estercolado, 31.05.2016.

Citada de Amurrio, Tertanga, VN9758, 400 m (Patino \& Valencia, 1989) y de Monte Santiago (BU) por Alejandre et al. (2006). Tercera cita de Sierra Sálvada y su entorno.

\section{Rhinanthus minor L.}

(VI) Amurrio, Bigandi, WN0360, 670 m, grupo de 12 tallos florecidos en el borde de una charca, 29.05.2015 (Fig.2).

Plantas con corolas inferiores a $16 \mathrm{~mm}$ de longitud y tubo recto (Sáez, 2009). Primera cita en la comunidad autónoma del País Vasco, al menos mientras no se confirmen las citas desechadas por Aseginolaza et al. (1985). 


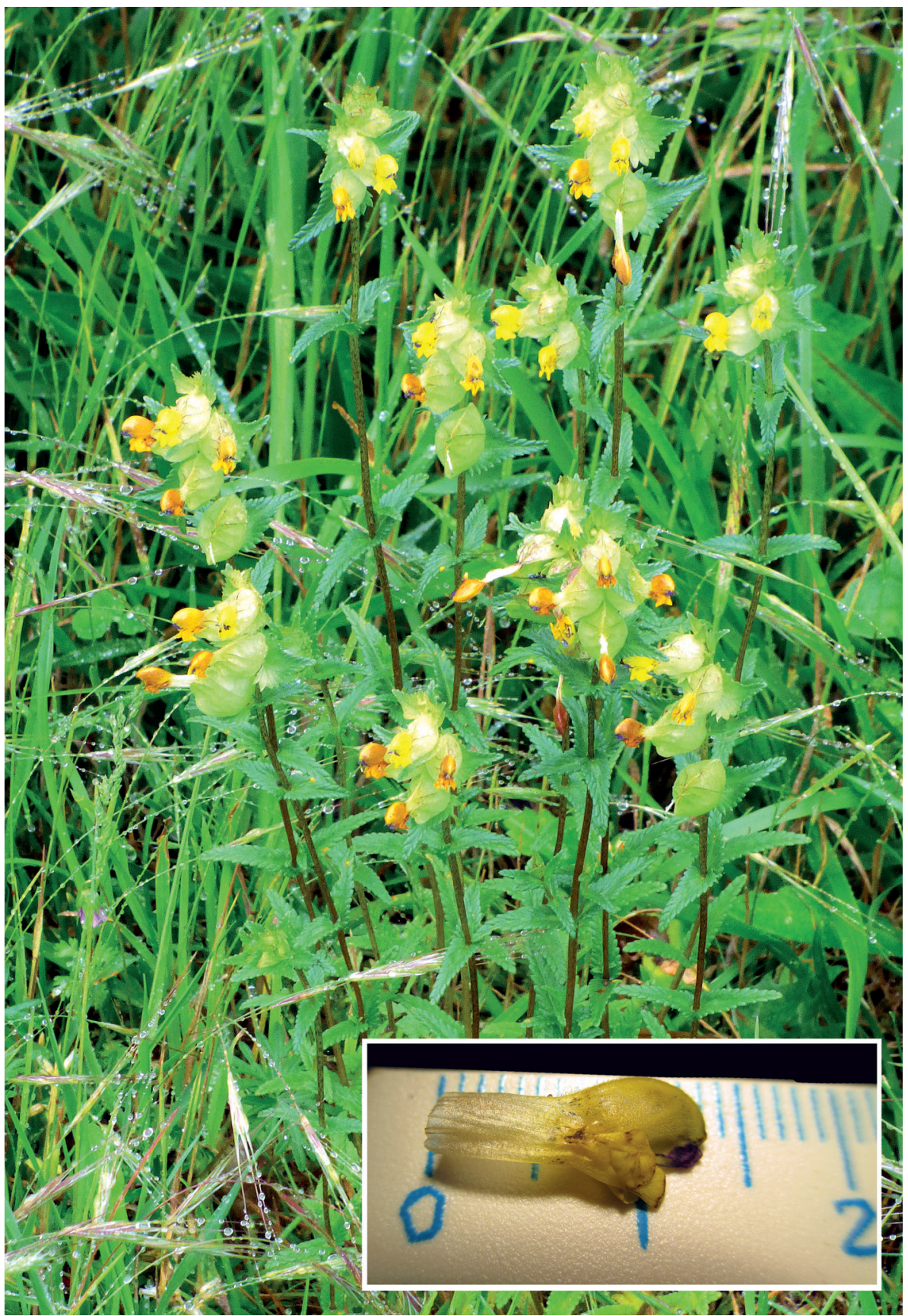

Fig. 2.- Rhinanthus minor, fotografiada en Amurrio.

Fig. 2.- Rhinanthus minor, photographed in Amurrio. 


\section{Sagina saginoides (L.) H. Karst.}

(BI) Zeanuri, Igiñiber, WN1864, 1.350 m, pasto montano sobre calizas, 14.06.2014.

En Bizkaia y Macizo del Gorbeia solo está citada de Zeanuri, monte Aldamin, WN1865, 1.250 m (Aseginolaza et al., 1985). Segunda localidad, cercana a la ya conocida.

\section{Sorbus torminalis (L.) Crantz}

(BI) Orduña, Lendoño de Abajo, VN9862, 420 m, dos arbolitos, 22.11.2016.

Primera cita en Sierra Sálvada y su entorno.

\section{Stegnogramma pozoi (Lag.) K. Iwats.}

(BI) Zaratamo, Upoerreka, WN1081 y WN1082, 240-300 m, bordes de arroyo, 29.03.2015.

(BI) Arrankudiaga, Goikogane, WN0380, 530 m, borde de arroyo, 25.04.2015.

(BI) Arrankudiaga, Anibarro erreka, WN0383, 400 m, borde de arroyo, 18.11.2015.

(BI) Arakaldo, Zileku erreka, WN0677, 250 m, borde de arroyo, 17.02.2016.

Poblaciones en nuevos arroyos afluentes del río Nervión (véase Barredo, 1996 y Pérez de Ana, 2014).

\section{Tragopogon porrifolius L.}

(VI) Amurrio, Tertanga, VN9758, 400 m, borde de camino, 16.06.2014.

(BI) Orduña, La Rondina VN9859, 300 m, borde de camino, 14.05.2015.

Plantas con lígulas violáceo-purpúreas, involucro con 8 brácteas y pedúnculos engrosados en la antesis (Díaz de la Guardia \& Súarez Santiago, 2009). Antiguamente cultivada. Aseginolaza et al. (1985) no la encontraron en el País Vasco. Llorente et al. (2009) la citaron en Urduliz (Bi). Segunda y tercera citas en la comunidad autónoma del País Vasco.

\section{Valeriana officinalis $\mathrm{L}$.}

(VI) Ayala, Izoria, WN9666, 270 m, 14 tallos florecidos en cuneta herbosa y sombreada, 14.05.2015.

(VI) Ayala, monte Ungino, VN9164, 720 m, 3 plantas en borde de marojal, 31.05.2016.

Cuarta y quinta localidad de Sierra Sálvada (véase Pérez de Ana, 2007-2008 y 2009-2010).

\section{Veronica anagalloides Guss. subsp. anagalloides}

(VI) Amurrio, Bigandi, WN0360, 670 m, borde de charca, 12.06.2014.

Primera cita en Sierra Sálvada y su entorno (Aseginolaza et al., 1985). 


\section{Bibliografía}

- Aedo, C. 2013. Allium. En: Flora iberica. E. Rico, M.B. Crespo, A. Quintanar, A. Herrero, C. Aedo (Ed.): 220-273. Real Jardín Botánico, CSIC. Madrid.

- Aizpuru, I., Aparicio, J. M., Aperribay, J. A., Aseginolaza, C., Elorza, J., Garin, F., Patino, S., Pérez Dacosta, J.M., Pérez de Ana, J.M., Uribe-Echebarría, P.M., Urrutia, P., Valencia, J., Vivant, J. (1996). Contribuciones al conocimiento de la flora del País Vasco. An. Jar. Botánico Madrid 54: 419-435.

- Aizpuru, I., Aperribay, J. A., Balda, A., Garin, F., Lorda, M., Olariaga, I., Terés, J., Vivant, J. 2003. Contribuciones al conocimiento de la flora del País Vasco (V). Munibe Cienc. Nat. 54: 39-74.

- Aizpuru, I., Aseginolaza, C., Garin, F., Vivant, J. 1998. Contribuciones al conocimiento de la flora del País Vasco, III. Munibe Cienc. Nat. 50: 7-19.

- Alejandre Sáenz, J.A., García-López, J.M., Mateo Sanz, G. (Ed.) 2006. Atlas de la flora vascular silvestre de Burgos. Junta de Castilla y León y Caja Rural de Burgos.

- Argüelles, J.M., Carlón, L., Gómez Casares, G., González del Valle, J.M., Laínz, M., Moreno Moral, G., Sánchez Pedraja, Ó. 2004-2005. Contribuciones al conocimiento de la flora cantábrica, VII. Bol. Cienc. Nat. R.I.D.E.A. 49: 147-193.

- Aseginolaza Iparragirre, C., Gómez García, D., Lizaur Sukia, X., Montserrat Martí, G., Morante Serrano, G., Salaverría Monfort, M.R., Uribe-Echebarría Díaz, P.M., Alejandre Sáenz, J.A. 1985. Catálogo Florístico de Álava, Vizcaya y Guipúzcoa. Servicio Central de Publicaciones del Gobierno Vasco. Vitoria-Gasteiz.

- Barredo Pérez, J.J. 1996. Nuevos datos sobre la distribución y el hábitat de algunas plantas termófilas oceánicas en el tramo oriental de la Cornisa Cantábrica. Munibe Cienc. Nat. 48: 39-48.

- Barredo Pérez, J.J. 2005. Hallazgo de plantas nuevas o raras en Vizcaya. Munibe Cienc. Nat. 56: 49-54.

- Campos, J. A., Berastegi, A., Darquistade, A. 2002. Sobre algunas plantas poco conocidas del País Vasco y zonas limítrofes. Estud. Museo Cienc. Nat. Álava, 17: 125-130.

- Crespo, M.B. 2005. Epipactis. En: Aedo, C., Herrero, A. (Ed.). Flora iber. 21: 22-54.

- Díaz de la Guardia, C., Suárez Santiago, V.N. 2009. Tragopogon. En: Flora Vascular de Andalucía Oriental 4. G. Blanca, B.M. Cueto, C. Fernández López, C. Morales Torres (Ed.): 311-314. Consejeria de Medio Ambiente, Junta de Andalucia. Sevilla.

- Gandoger, J.M. 1917. Catalogue des plantes récoltées en Espagne et en Portugal pendant mes voyages de 1894 à 1912. Hermann, L'homme, Masson libraires. Paris.

- Guinea, E. 1953. Geografía botánica de Santander. Diputación Provincial de Santander. Santander.

- Hermosilla, C.E., Sabando, J. 1993. Notas sobre orquídeas. Estud. Museo Cienc. Nat. Álava 8: 73-84.

- Hermosilla, C. E., Sabando, J. 1995. Notas sobre orquídeas (III). Estud. Museo Cienc. Nat. Álava 10-11: 141-194.

- Hermosilla, C.E., Sabando, J. 1995. Notas sobre orquídeas (V). Estud. Mus. Cienc. Nat. Álava 13: 123-156. 
- Luceño, M. 2007. Carex. En: Flora iberica 18, J. Castroviejo, M. Luceño, A. Galán, P. Jiménez Mejías, F. Cabezas, L. Medina, L. (Ed.). : 109-250. Real Jardín Botánico, CSIC. Madrid.

- Llamas, F., Acedo, C., Penas, A., Pérez Morales, C. 2002. Una nueva subespecie de Festuca paniculata en la Cordillera Cantábrica. Lagascalia 22: 119-129.

- Llorente Rodrigo, A., Cadiñanos Aguirre, J.A., Fidalgo Prieto, E. 2009. Aportaciones a la flora vascular de Vizcaya, Guipúzcoa y Cantabria. Munibe Cienc. Nat. 57: 47-65.

- Onaindia Olalde, M. 1986. Ecología vegetal de las Encartaciones y Macizo del Gorbea -Vizcaya. Servicio Editorial de la Universidad del País Vasco. Bilbao.

- Patino, S., Valencia, J. 1989. Nuevas aportaciones al catálogo florístico de la Comunidad Autónoma Vasca. Estud. Mus. Cienc. Nat. Álava 4: 77-84.

- Patino, S., Valencia, J. Miguel, E., Prieto, A., Elorza, J., Oyanarte, T., Toral, R., Otxoa, F., Díaz. E. 2009-2010. Notas corológicas sobre la flora vascular del País Vasco y aledaños (XII). Estudios del Museo de Ciencias Naturales de Álava, 23: 91-111.

- Pérez de Ana, J. M. 2007-2008. Nuevas citas de flora amenazada y rara en Sierra Salvada. Estud. Mus. Cienc. Nat. Álava 22: 85-100.

- Pérez de Ana, J. M. 2009-2010. Nuevas citas de flora amenazada y rara en Sierra Salvada, II. Estud. Mus. Cienc. Nat. Álava 23: 61-66.

- Pérez de Ana, J.M. 2014. Nuevas citas de flora amenazada y rara en el País Vasco. Munibe Cienc. Nat. 62: 103-117.

- Pérez-Morales, C., Penas, Á., Llamas, F., Acedo, C. 1994. Doronicum pubescens sp. nov. Lazaroa 14: 5-12.

- Sáez, L. 2009. Rhinanthus. En: Flora iberica 13. C. Benedí, E. Rico, J. Güemes, A. Herrero (Ed.). : 531-536. Real Jardín Botánico, CSIC. Madrid.

- Silván, F., Loidi, J., Campos, J. A., Darquistade, A., Biurrun, I. García, I., Olano, J.M., IKT, S.A. 2000. Flora y Vegetación del Parque Natural de Valderejo, Vol II. Diputación Foral de Álava. Departamento de Agricultura. Inédito.

- Talavera, S., Casimiro-Rodríguez, R., Molina, J. A., Pizarro, J. M. 2010. Baldellia. En: Flora iberica 17. S. Talavera, M.J. Gallego, C. Romero Zarco, A. Herrero (Ed.): 11-18. Real Jardín Botánico, CSIC. Madrid.

- Uribe-Echebarría, P.M. 1981. Algunas plantas que viven en Álava. An. Jard. Botánico Madrid 38(1): 309-313.

- Uribe-Echebarría, P.M. 1982. Pteridófitos alaveses. Collectanea Botanica 13(1): 101-117.

- Uribe-Echebarría, P.M. 2001. Notas corológicas sobre la flora vascular del País Vasco y aledanos (X). Estud. Mus. Cienc. Nat. Álava 16: 93-102.

- Fecha de recepción/Date of reception: 01-12-2016

- Fecha de aceptación/Date of acceptance: 10-02-2017

Editor Asociado/Associate editor: Ricardo Ibáñez 